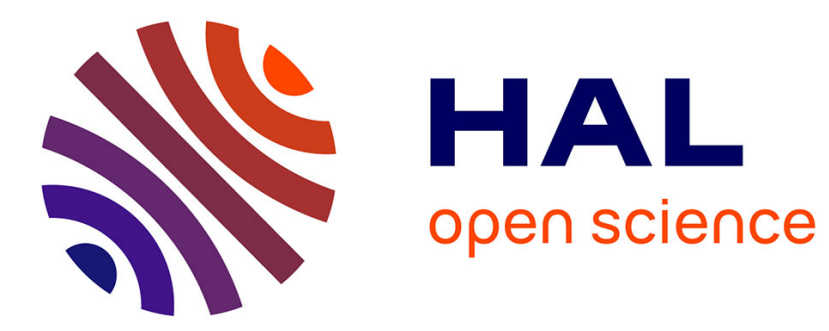

\title{
Blind cooperative diversity using Distributed Space-Time Coding in Block Fading Channels
}

Kamel Tourki, Mohamed-Slim Alouini, Luc Deneire

\section{To cite this version:}

Kamel Tourki, Mohamed-Slim Alouini, Luc Deneire. Blind cooperative diversity using Distributed Space-Time Coding in Block Fading Channels. IEEE International Conference on Communications, May 2008, Beijing, China. pp.1. hal-00224226

\section{HAL Id: hal-00224226 \\ https://hal.science/hal-00224226}

Submitted on 30 Jan 2008

HAL is a multi-disciplinary open access archive for the deposit and dissemination of scientific research documents, whether they are published or not. The documents may come from teaching and research institutions in France or abroad, or from public or private research centers.
L'archive ouverte pluridisciplinaire $\mathbf{H A L}$, est destinée au dépôt et à la diffusion de documents scientifiques de niveau recherche, publiés ou non, émanant des établissements d'enseignement et de recherche français ou étrangers, des laboratoires publics ou privés. 


\section{Blind cooperative diversity using Distributed Space-Time Coding in Block Fading Channels}

\author{
Kamel Tourki \\ Laboratoire I3S CNRS UNSA \\ 2000, rte des Lucioles BP 121 \\ 06903 Sophia Antipolis Cedex \\ Email: tourki@i3s.unice.fr
}

\author{
Mohamed-Slim Alouini \\ Texas A\&M University at Qatar \\ Education city PO Box 23874 \\ Doha, Qatar \\ Email: Alouini@qatar.tamu.edu
}

\author{
Luc Deneire \\ Laboratoire I3S CNRS UNSA \\ 2000, rte des Lucioles BP 121 \\ 06903 Sophia Antipolis Cedex \\ Email: deneire@i3s.unice.fr
}

\begin{abstract}
Mobile users with single antennas can still take advantage of spatial diversity through cooperative space-time encoded transmission. In this paper, we consider a scheme in which the relay chooses to cooperate only if the source-relay channel is of an acceptable quality and we evaluate the usefulness of relaying when the source acts blindly and ignores the decision of the relay if it may cooperate or not. In our study, we consider a regenerative relay in which the decision to cooperate is based on a signal-to-noise ratio (SNR) threshold and consider the effect of the possible erroneously detected and transmitted data at the relay. We derive the end-to-end bit-error rate (BER) for binary phase-shift keying modulation and look at two power allocation strategies between the source and the relay in order to minimize the end-to-end BER at the destination for a high SNR. Some selected performance results show that computer simulations based results coincide with our analytical results.
\end{abstract}

\section{INTRODUCTION}

In many wireless applications, wireless users may not be able to support multiple antennas due to size, complexity, power, or other constraints. The wireless medium brings along its unique challenges such as fading and multiuser interference, which can be mitigated with cooperative diversity [1]-[4]. Emamian and Kaveh proposed the cooperation as solution for combating shadowing [5] and Sendonaris et al. showed that cooperation among users can enlarge the capacity region of an uplink multiuser channel [6]. In traditional cooperative diversity setups, a user is unilaterally designated to act as a relay for the benefit of another one, at least for a given period of time. In certain scenarios, the relay is an actual component of the infrastructure with no own data to be delivered to the network [7]-[11]. Therefore most of these systems use the Decode-and-Forward (DF) or regenerative protocol for cooperation when the relay decodes perfectly the message sent by the source [12]-[14]. Thereby the relay uses feedback to inform the source in order to cooperate, which may be a restrictive condition. We try to overcome these restrictions by using distributed space-time coding (DSTBC) which improves bandwidth efficiency on top of diversity.

A major challenge in distributed cooperative transmissions is to find a way to coordinate the relay transmissions without requiring extra control information (e.g. feedback) overhead, which would reduce part of the gain. The decision to relay can be taken using a cyclic redundancy check (CRC) codes [15] or using a signal-to-noise ratio (SNR) threshold at the relay as in [16].

We choose DF protocol for communicating in our setup due to the advantages cited in [9] when the relay is near to the source, and we use an SNR threshold to decide if the relay may decode or not. Hence the transmission must be done in two phases. In the first phase, the source communicates its information to the relay. In the second phase and depending on the relay decision, the destination receives from the source and the relay or only from the source. Therefore we consider resource control in the form of power allocation by the source across the two phases.

As expected, the relay may retransmit an erroneously decoded message. Using Alamouti scheme [17], we determine the end-to-end bit error rate (BER) expression for the binary phase shift keying (BPSK) modulation taking the relay error propagation into account. There will be a compromise to strike between the transmit power of the source in the first phase and the decoding threshold SNR at the relay in the second phase.

\section{A. Related Works}

Other contributions on selective decode and forward cooperative communication under imperfect regeneration are presented in [16], [18]. In [16], the source broadcasts its message to relay and destination. In the second phase, if the relay has decided to forward, it retransmits its received signal to the destination. This scheme is well known as time repetition coding in which the destination combines the received signal from the source and relay. Otherwise, if the relay has not decided to decode, it remains silent. This induces a rate loss with respect to non cooperative communication because the data is transmitted from different points in space, during different time slots. In [18], the relay is allowed to make errors and the authors opted for DSTC with OFDM for a block fading channel. Therefore they proposed an optimal maximum likelihood (ML) decoder which exploits the knowledge of the error statistics at the relay and a suboptimal decoder when this knowledge is not available.

The remainder of this paper is organized as follows. In section II, we describe the system model and our proposed blind cooperation mode of opration. In section III the end-to-end BER expression is derived and the optimal power allocation 
TABLE I

TRANSMISSION SCHEME IN PHASE II.

\begin{tabular}{|c||c|c|}
\hline & $T_{1}$ & $T_{2}$ \\
\hline source & $\mathbf{s}(n)$ & $\mathbf{s}(n+1)$ \\
relay (if $\left.\gamma>\gamma_{0}\right)$ & $-\widetilde{\mathbf{s}}^{*}(n+1)$ & $\widetilde{\mathbf{s}}^{*}(n)$ \\
\hline
\end{tabular}

and decoding threshold SNR are determined. Finally, some selected simulation results are depicted in section IV while some concluding remarks are given in section V.

\section{SYSTEM MODEL}

In this section we describe the distributed Alamouti scheme and we note that only one nearby relay is targeted to cooperate. The system model obeys to the topology depicted in Fig. 1 and we restrict our model to three nodes. We assume that each terminal is equipped with one antenna. As depicted in Fig. 1, the transmission is done in two phases, and we must balance the need of resources. The source allocates a power fraction equal to $\alpha P$ for its transmission to the relay in the first phase, and the remaining power is dedicated to the second phase. We denote $h_{s r}, h_{s d}$ and $h_{r d}$ as the coefficients of the channels between the source (S) and the relay (R), the source and the destination (D) and the relay and the destination, respectively.

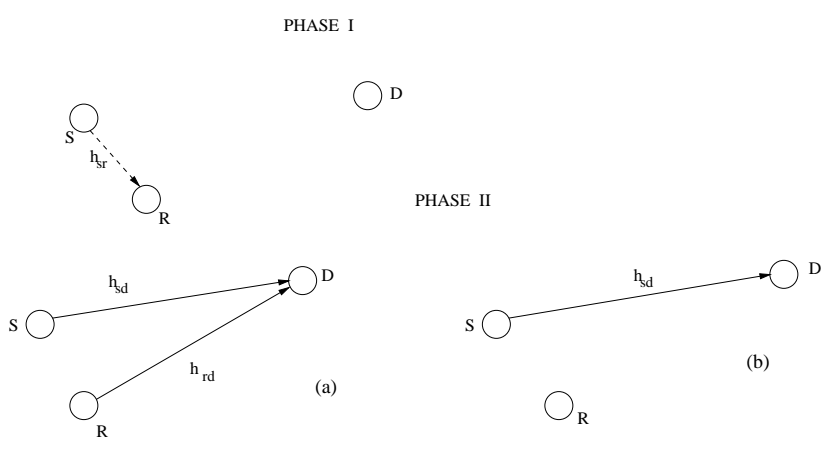

Fig. 1. Cooperative communication system.

\section{A. Blind cooperation}

We describe the proposed transmission protocol which is a time division duplex (TDD) scheme and summarized by Table I. Each frame is subdivided in two consecutive BPSK Nsize information symbol blocks $\mathbf{s}(n)$ and $\mathbf{s}(n+1)$ to be transmitted in two phases. In the first phase, the source broadcasts $[\mathbf{s}(n) ; \mathbf{s}(n+1)]$ using only the power fraction $\alpha P$. Within this phase, the destination does not consider the received data, but intuitively, we can expect that this power fraction must be as small as possible in order to save more power for the next phase. Therefore in phase I only the relay is assumed to receive the transmitted signal and the $\mathrm{N}$-size signal vectors $\mathbf{y}_{\mathbf{r}}(i)$ received are:

$$
\mathbf{y}_{\mathbf{r}}(i)=\sqrt{\alpha P} h_{s r} \mathbf{s}(i)+\mathbf{n}_{\mathbf{r}}(i), \quad i=n, n+1
$$

where $\mathbf{n}_{\mathbf{r}}(i)$ is the additive-noise vector at the relay with a covariance matrix $N_{0} \mathbf{I}_{N}$ and we denote $\gamma_{r}=\frac{\alpha P\left|h_{s r}\right|^{2}}{N_{0}}$ the received SNR at the relay.

In the second phase, the source retransmits $[\mathbf{s}(n) ; \mathbf{s}(n+1)]$ using the power fraction $\alpha_{1} P$. The relay transmission is conditioned by its the received SNR $\gamma_{r} ; 1$ ) If it exceeds the decoding threshold SNR $\gamma_{0}$, the relay decodes the data as $[\widetilde{\mathbf{s}}(n) ; \widetilde{\mathbf{s}}(n+1)]$. Hence in the following block, the relay sends $\left[-\widetilde{\mathbf{s}}^{*}(n+1) ; \widetilde{\mathbf{s}}^{*}(n)\right]$ using the power fraction $\alpha_{2} P$ and the destination sees a distributed space-time code as

$$
\begin{aligned}
\underbrace{\left[\begin{array}{c}
\mathbf{y}_{\mathbf{d}}(n) \\
\mathbf{y}_{\mathbf{d}}(n+1)
\end{array}\right]}_{\mathbf{y}_{d}}= & \sqrt{P} \underbrace{\left[\begin{array}{cc}
\sqrt{\alpha_{1}} \mathbf{s}(n) & -\sqrt{\alpha_{2}} \widetilde{\mathbf{s}}^{*}(n+1) \\
\sqrt{\alpha_{1}} \mathbf{s}(n+1) & \sqrt{\alpha_{2}} \widetilde{\mathbf{s}}^{*}(n)
\end{array}\right]}_{\mathbf{S}} \underbrace{\left[\begin{array}{c}
h_{s d} \\
h_{r d}
\end{array}\right]}_{\mathbf{h}} \\
& +\underbrace{\left[\begin{array}{c}
\mathbf{n}_{\mathbf{d}}(n) \\
\mathbf{n}_{\mathbf{d}}(n+1)
\end{array}\right]}_{\mathbf{n}_{d}}
\end{aligned}
$$

where $\mathbf{n}_{d}$ is the additive noise vector at the destination with covariance matrix $N_{0} \mathbf{I}_{2 N}$.

Otherwise, 2) if $\gamma_{r}<\gamma_{0}$, the source which ignores the relay decision is sending and $\mathrm{D}$ receives

$$
\mathbf{y}_{\mathbf{d}}(i)=\sqrt{\alpha_{1} P} h_{s d} \mathbf{s}(i)+\mathbf{n}_{\mathbf{d}}(i), \quad i=n, n+1
$$

where $\mathbf{n}_{\mathbf{d}}(i)$ is the additive-noise vectors at $\mathrm{D}$ with a covariance matrix $N_{0} \mathbf{I}_{N}$. We note that there is no feedback from the relay to the source which transmits blindly in phase II. But even if the source and the relay are synchronized to transmit, their packets might arrive asynchronous at the destination. We can deal with this problem using the algorithm of [19]. But focusing only on the transmission protocol, we can assume that the signals reach the destination in the same time.

\section{B. Detection procedure}

Each transmitting node use a CDMA code which is implemented as a training sequence. From a certain code $\underline{c}$ we can form two orthogonal codes $\mathbf{c}_{1}=[\underline{c} ; \underline{c}]$ and $\mathbf{c}_{2}=[-\underline{c} ; \underline{c}]$. The source will use $\mathbf{c}_{1}$ and if the relay decide to cooperate, it will use $\mathbf{c}_{2}$. The rank of the code matrix $\mathbf{C}=\left[\mathbf{c}_{1} \mathbf{c}_{2}\right]$ gives us the number of cooperating nodes and the destination will decide which decoding technique to apply.

We can opt for another technique; if the relay decide to cooperate, it transmits a specific bit $b=1$ to inform the destination that it will send. Otherwise, when the destination never receives this bit, it supposes that only source is transmitting.

\section{Performance AnAlysis}

In the traditional DF protocol, the relay cooperates only when it decodes perfectly the message. Therefore there is no risk of error propagation by the relay, and in cooperative scheme, the distributed nodes use a space time code as in MIMO systems. But in our scheme, depending on the SNR threshold level $\gamma_{0}$, the situation changes because the relay can retransmit an erroneously decoded message. Therefore we need to determine the end-to-end performance of this system which is expressed as

$$
P_{e, s y s}=P_{d e c} P_{e}^{1}+\left(1-P_{d e c}\right) P_{e, d}
$$


where $P_{d e c}$ is the relay decoding probability, (see Appendix. A) and $P_{e, d}$ is the probability of error for the direct communication between the source and the destination. In (4), $P_{e}^{1}$ is the error probability at $\mathrm{D}$ when $\mathrm{S}$ and $\mathrm{R}$ cooperate.

When $\gamma_{r}>\gamma_{0}$, we enumerate these cases:

- The relay decodes with errors the received message, and this event has a probability of $P_{e}^{s, r}$.

- The relay decodes perfectly the received message with probability of $1-P_{e}^{s, r}$.

Therefore $P_{e}^{1}$ is derived as

$$
P_{e}^{1}=0.5 \times P_{e}^{s, r}+\left(1-P_{e}^{s, r}\right) P_{e}^{2}
$$

where $P_{e}^{2}$ is the error probability for the 2 by 1 Alamouti scheme which depends on the network architecture, and 0.5 is the largest error probability when the Alamouti scheme orthogonality is broken. $P_{e}^{s, r}$ is derived as (see Appendix. B for the proof):

$$
\begin{aligned}
P_{e}^{s, r}= & a Q\left(\sqrt{b \gamma_{0}}\right)-a \exp \left(\frac{\gamma_{0}}{\bar{\gamma}}\right) \sqrt{\frac{1}{1+\frac{2}{b \bar{\gamma}}}} \\
& \times Q\left(\sqrt{2 \gamma_{0}\left(\frac{b}{2}+\frac{1}{\bar{\gamma}}\right)}\right)
\end{aligned}
$$

where $Q($.$) is the Marcum Q-function, a and \mathrm{b}$ depend on the modulation.

\section{A. Parameter optimization}

The most important parameters which control the proposed scheme are the power fraction $\alpha$ and the decoding threshold SNR at the relay $\gamma_{0}$. Both parameters must be chosen to satisfy:

$$
\left(\alpha_{m}, \gamma_{0 m}\right)=\arg \min P_{e, s y s}
$$

where $\alpha \in] 0,1\left[\right.$ and $\gamma_{0} \in \mathbb{R}$. Given the complicated form of $P_{e, s y s}$, it is evident that this optimization can not be conducted analytically in a straightforward fashion. Therefore we will look in what follows for the optimum parameters based on some numerical results.

\section{Simulation Results}

\section{A. Network Geometry}

We anticipate that cooperation will perform differently as function of the positions of the users with respect to destination. Hence we study two different network geometries, denoted by symmetric network (SN) (see Fig. 1) and asymmetric or linear network (LN) (see Fig. 2). In the linear (LN) case, we model the path-loss, i.e. the mean channel powers $\sigma_{h}^{2}$, as a function of the relative relay position $r$ by

$$
\sigma_{s d}^{2}=1, \sigma_{s r}^{2}=d^{-\nu}, \sigma_{r d}^{2}=(1-d)^{-\nu}
$$

where $\nu$ is the path loss exponent and $0<d=d_{s r}<1$. The distances are normalized by the distance $d_{s d}$. In these coordinates, the source can be located at $(0,0)$, the destination can be located at $(1,0)$, without loss of generality, and the relay is located at $(\mathrm{d}, 0)[20]$. In the symmetric network $(\mathrm{SN}), h_{s d}$

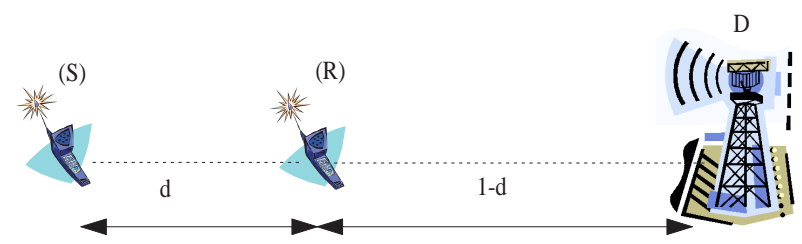

Fig. 2. Asymmetric (or linear) network.

and $h_{r d}$ are drawn with same unit-variance (equal sub channel gains), but considering source and relay are close together, we set $\sigma_{s r}^{2}=16$.

\section{B. Simulation Results}

In this section, we evaluate the performance of our scheme in terms of end-to-end BER at the destination as function of SNR $=P / N_{0}$ for a BPSK modulation. We report results for $\nu=4$, a block length $N=50$, and we model all channels as Rayleigh block flat fading with additive white Gaussian noise. Figs. 3-5 show the end-to-end performance of our scheme with optimized power allocation, compared with the non cooperative system and MISO system respectively. In order to make a fair comparison between different schemes, we enforce all systems to transmit with the same overall power. As mentioned before, $P_{e}^{2}$ depends on the network architecture.

a) Equal sub-channel gains $(S N)$ : For equal sub-channel gains $\gamma$, the moment generating function (MGF) of the instantaneously experienced SNR $\rho$ for a system with $t$ transmit antennas, $r$ receive antennas and $\lambda$ is the channel energy, can be expressed as [21]

$$
\phi_{\rho}(s)=\frac{1}{(1-\rho \times s)^{u}}
$$

where $R$ is the transmission rate, $u=t \times r$ and

$$
\rho=\frac{1}{R} \frac{\lambda}{t} \frac{S}{N}=\frac{\lambda}{t} \frac{E_{s}}{N_{0}}=\log _{2}(M) \frac{\lambda}{t} \frac{P}{N_{0}}
$$

where $E_{s}$ is the energy per symbol, $P$ the energy per bit and $M=2$ for BPSK modulation. We considered BPSK Alamouti scheme therefore $R=1, N=N_{0}$ and $\frac{S}{N}=\frac{P}{N_{0}}$. The analysis in [13], [14], [22] allows us to express the BER for BPSK modulation in closed form as

$$
\begin{aligned}
P_{t, r}\left(R, \gamma, \frac{S}{N}\right)= & \phi_{\frac{1}{R} \frac{\lambda}{t} \frac{S}{N}}(-1)\left[\frac{1}{2 \sqrt{\pi}} \frac{\Gamma(u+1 / 2)}{\Gamma(u+1)}\right] \times \\
& { }_{2} F_{1}\left(u, 1 / 2 ; u+1 ;\left(1+\frac{1}{R} \frac{\gamma}{t} \frac{S}{N}\right)^{-1}\right)(11)
\end{aligned}
$$

where ${ }_{2} F_{1}(a, b ; c ; x)$ is the Gauss hypergeometric function with 2 parameters of type 1 and 1 parameter of type 2 .

b) Unequal Sub-Channel Gains (LN): For unequal subchannel gains, the MGF cam be shown to be given by

$$
\phi_{\frac{1}{R} \frac{\lambda}{t} \frac{S}{N}}(s)=\sum_{i=1}^{u} K_{i} \phi_{\frac{1}{R} \frac{\lambda_{i}}{t} \frac{S}{N}}(s)
$$


TABLE II

OPTIMIZED PARAMETERS AT HIGH SNR $(30 d B)$.

\begin{tabular}{|c||c||c|c|}
\hline System & $S N$ & $L N, d=0.1$ & $L N, d=0.5$ \\
\hline$\alpha_{m}$ & 0.227 & 0.017 & 0.44 \\
$\gamma_{0 m}$ & 6.57 & 6.78 & 6.28 \\
\hline
\end{tabular}

with constants $K_{i}$ [13], [23] (See appendix C)

$$
K_{i}=\prod_{i^{\prime}=1, i^{\prime} \neq i}^{u} \frac{\overline{\gamma_{i}}}{\overline{\gamma_{i}}-\overline{\gamma_{i^{\prime}}}}
$$

where $\bar{\gamma}_{i}$ is the average channel gain of the $i^{\text {th }}$ path. This allows us to derive the expression of BER in closed form where all the channel gains differ. The error rate can be expressed as

$$
P_{u c g}(u)(e)=\sum_{i=1}^{u} K_{i} P_{t, r}\left(R, \bar{\gamma}_{i}, \frac{S}{N}\right)
$$

1) Equal transmit power in phase II: We set $\alpha_{1}=\alpha_{2}=$ $(1-\alpha) / 2^{1}$, and we determine the optimum variables in Eq. (7) at high SNR. We consider an overall transmit power $P$. Thereby, we must have $\alpha+\alpha_{1}+\alpha_{2}=1$. But with a blind source behavior, we note that the overall power will be less than $P$ when the relay decide to not cooperate because $\alpha_{2}=0$. The parameter optimization results for the SN and $\mathrm{LN}$ architectures are derived numerically at a high SNR and are collected in Table. II.

In the symmetric network, Fig. 3 shows that our scheme achieves full diversity and the influence of the distributed STBC with optimized parameters $\left(\alpha_{m}, \gamma_{0 m}\right)=(0.227,6.57)$ is small $(2 \mathrm{~dB})$ wrt the MISO system in which each antenna transmits with a power equal to $P / 2$. But the full diversity order performance will be saved for some appropriate variations on the parameters when we choose $(0.1,8)$. When we take a lower threshold SNR level $\gamma_{0}=0 d B$, we observe a little enhancement in the end-to-end performance for a low SNR, but we loose the full diversity order. Fig. 4 and 5 show the simulation results for a linear network when the relay is located between the source and the destination at $(0.1,0)$ and $(0.5,0)$ respectively. The gains due to the optimized power allocation in the cooperation are clearly more significant when the relay is close to the source $(d=0.1)$. At this situation, it is clear that our scheme never performs worse than a non cooperative scheme. Thereby, our results confirm the fact that the DF protocol maximizes the capacity when the relay is near to the source [9]. We note that the influence of the distributed STBC with optimized parameters is more significant than the $\mathrm{SN}$ case, and it is interesting to note that all simulation results are in agreement with our analysis-based results.

2) Unequal transmit power in phase II: Dividing the remaining power for the second phase between the source and the relay, the power fraction $(1-\alpha) P / 2$ will be lost if the relay decides to not cooperate. In order to overcome

\footnotetext{
${ }^{1} \alpha_{1}$ and $\alpha_{2}$ can be optimized based on channel gains of the links between $\mathrm{S}$ and $\mathrm{D}$, and between $\mathrm{R}$ and $\mathrm{D}$.
}

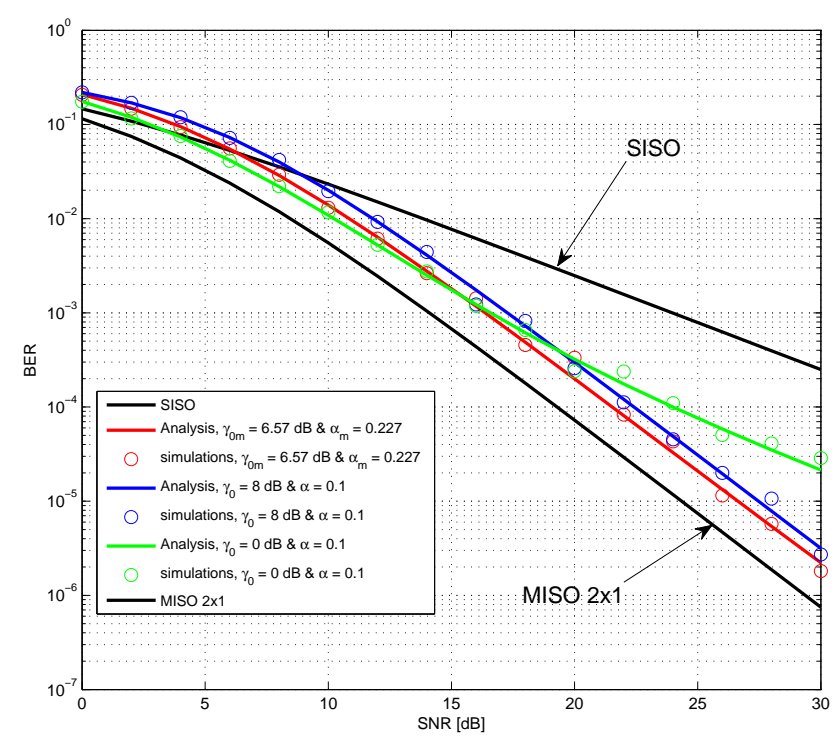

Fig. 3. Performance results of the symmetric network (SN) when the source and the relay use the same transmit power $\alpha_{1}=\alpha_{2}=(1-\alpha) / 2$.

this unbalanced allocation, we take another power allocation strategy for which the source and the relay have independent power constraints. In the second phase, the source and the relay transmit with respective power fractions ${ }^{2}$

$$
\left\{\begin{array}{l}
\alpha_{1}=(1-\alpha) \\
\alpha_{2}=1, \text { if } \quad \gamma_{r} \geq \gamma_{0}
\end{array}\right.
$$

In the symmetric network, Fig. 6 shows that our scheme achieves full diversity and the influence of the distributed STBC with optimized parameters $\left(\alpha_{m}, \gamma_{0 m}\right)=(0.413,6.92)$ is small with respect to the MISO system in which, each antenna transmits with a power equal to $P$. In this case, we note that the DSTC is never performs worse than non cooperative system (SISO), because for all SNR levels, the system uses at least a transmit power $P$ as the non cooperative system.

\section{CONCLUSions}

In this work, we studied the performance of a blind cooperation using a distributed STBC scheme, and we have shown that a minimized end-to-end BER is reached if the relay position is well chosen. If the relay is sufficiently near to the source, more power is saved to transmit the data from the source to the relay, and the error at the relay detector can be minimized with an appropriate choice of a predetermined cut-off threshold $\gamma_{0}$. Also we can note that with a closer relay we reduce the synchronization problem at the destination.

\section{APPENDIX}

In this appendix, we collect all the proofs.

\footnotetext{
${ }^{2}$ The source and relay may have different power constraints $P_{S}$ and $P_{R}$ where $P_{R}<P_{S}$.
} 


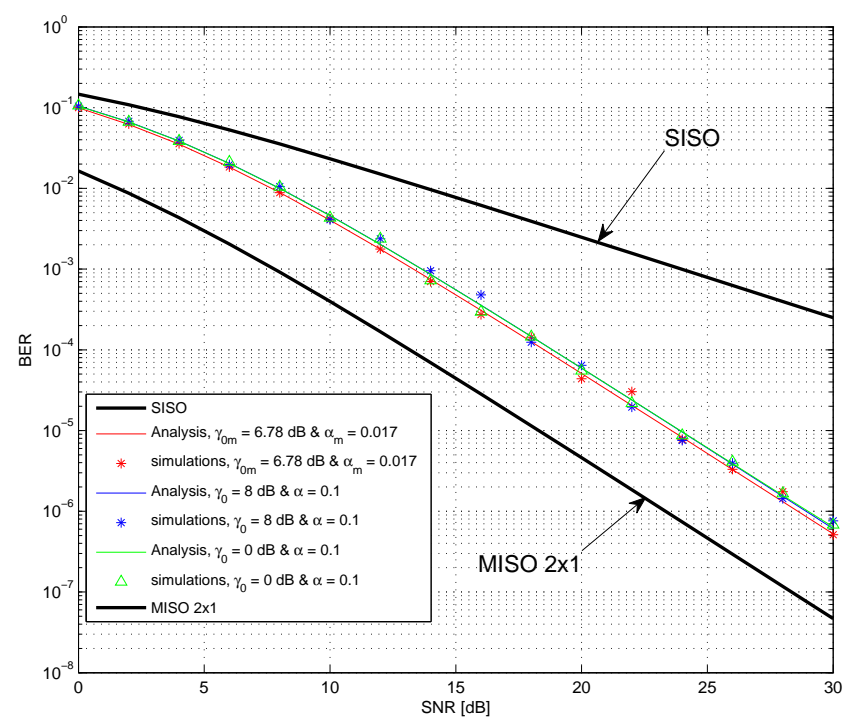

Fig. 4. Performance results of the linear network (LN), where the relay is near to the source $(\mathrm{d}=0.1)$ and both transmitting with the same power $(1-\alpha) / 2$.

\section{A. Decoding probability}

We consider the probability that the relay decodes the signal transmitted by the source. The relay decodes if its received SNR $\gamma$ is larger than the chosen SNR threshold $\gamma_{0}$.

$$
P_{\text {dec }}=P\left(\gamma>\gamma_{0}\right)
$$

The instantaneous SNR is determined by the fading channel power $\left|h_{s r}\right|^{2}$, the power fraction $\alpha$ allocated to the first phase, and the average SNR. Therefore,

$$
P_{d e c}=\int_{\frac{\gamma_{0}}{\alpha, \Gamma}}^{\infty} \frac{1}{\sigma_{s r}^{2}} \exp \left(-\frac{t}{\sigma_{s r}^{2}}\right) d t=\exp \left(-\frac{\gamma_{0}}{\sigma_{s r}^{2} \alpha \Gamma}\right)
$$

where $\Gamma=\frac{P}{N_{0}}$.

\section{B. Probability of Error at a decoding Relay}

We consider a special case of communication over a Rayleigh flat-fading channel where detection is performed only when the instantaneous SNR exceeds a threshold $\gamma_{0}$. The resulting pdf of the effective SNR is a clipped exponential function :

$$
p_{\gamma}^{\left(\gamma_{0}\right)}(\gamma)=\left\{\begin{array}{cc}
0 & \gamma<\gamma_{0} \\
\frac{1}{c} \frac{1}{\bar{\gamma}} \exp \left(-\frac{\gamma}{\bar{\gamma}}\right) & \gamma \geq \gamma_{0}
\end{array}\right.
$$

where $\bar{\gamma}$ is the mean SNR of the Rayleigh fading channel, and $c=\exp \left(-\gamma_{0} / \bar{\gamma}\right)$ is a normalizing constant ensuring unit area under the pdf. In the following, we determine the error probability for BPSK, which is given by

$$
\begin{aligned}
P_{e}^{s, r} & =\int P_{e}(\gamma) p_{\gamma}(\gamma) d \gamma \\
& =\int_{\gamma_{a}}^{\gamma_{b}} a Q(\sqrt{b \gamma}) p_{\gamma}(\gamma) d \gamma
\end{aligned}
$$

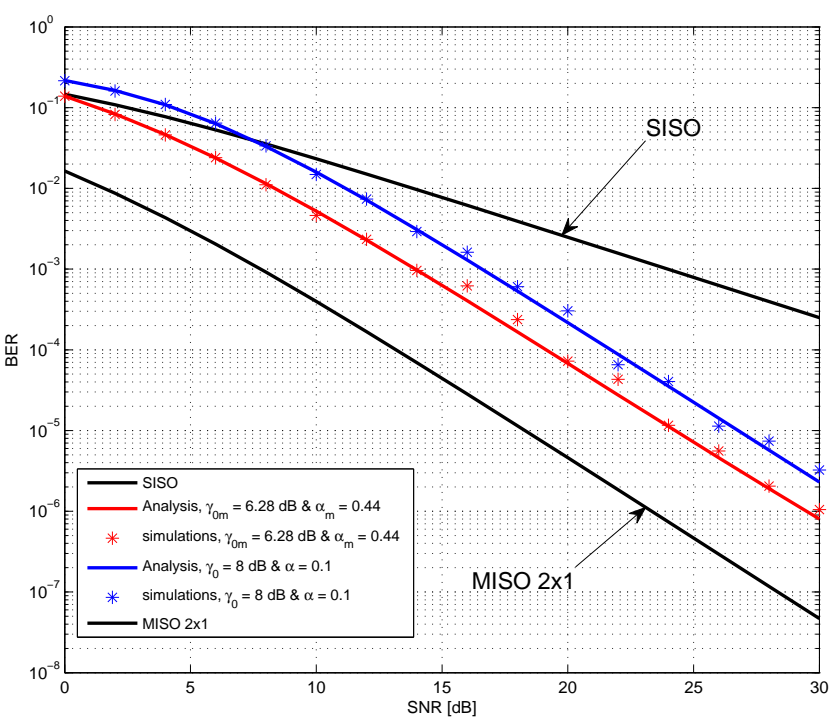

Fig. 5. Performance results of the linear system, where the relay is halfway between the source and the destination $(\mathrm{d}=0.5)$. Both use an equal transmitting power.

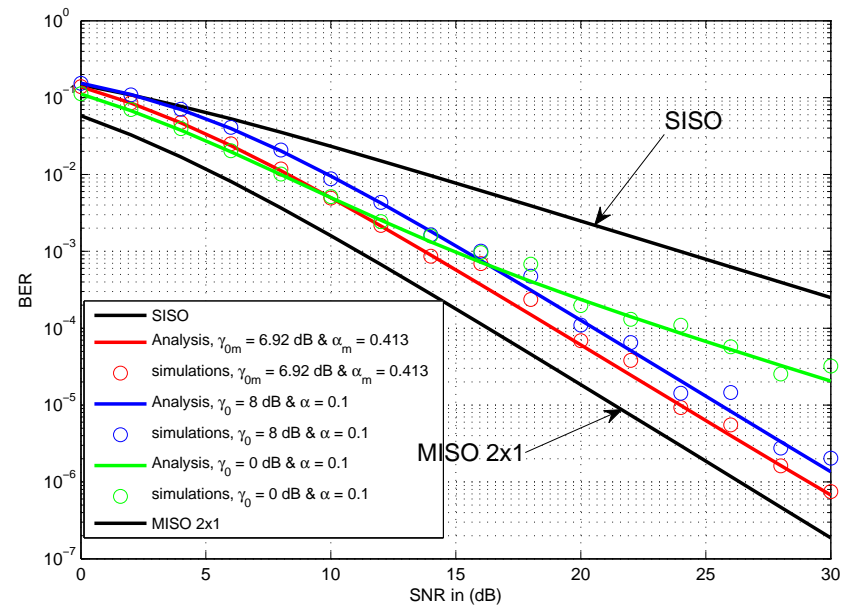

Fig. 6. Performance results of the symmetric network (SN) when the source allocates a transmit power $\alpha P$ in phase I and the remaining power in the second phase while the relay transmits with a power equal to $P$.

where $a=1, b=2$ and

$$
\begin{aligned}
Q(\sqrt{b \gamma}) & =\int_{\sqrt{b \gamma}}^{\infty} \frac{1}{\sqrt{2 \pi}} \exp \left(-\frac{t^{2}}{2}\right) d t \\
& =1-\int_{0}^{\sqrt{b \gamma}} \frac{1}{\sqrt{2 \pi}} \exp \left(-\frac{t^{2}}{2}\right) d t
\end{aligned}
$$

Introducing

$$
u(\gamma)=\int p_{\gamma}(\gamma) d \gamma
$$

we obtain by partial integration of (19)

$$
P_{e}^{s, r}=[u(\gamma) a Q(\sqrt{b \gamma})]_{\gamma_{a}}^{\gamma_{b}}-\int_{\gamma_{a}}^{\gamma_{b}} \frac{d}{d \gamma}(a Q(\sqrt{b \gamma})) u(\gamma) d \gamma
$$


Following the Leibnitz's rule for differentiation of integrals

$$
\begin{aligned}
\frac{\partial}{\partial x} \int_{f(x)}^{g(x)} F\left(x, x^{\prime}\right) d x^{\prime} & =F(x, g(x)) \frac{\partial g(x)}{\partial x}-F(x, f(x)) \frac{\partial f(x)}{\partial x} \\
& +\int_{f(x)}^{g(x)} \frac{\partial}{\partial x} F\left(x, x^{\prime}\right) d x^{\prime}
\end{aligned}
$$

With

$$
\left\{\begin{array}{c}
x^{\prime}=t \\
x=\gamma \\
g(\gamma)=\sqrt{b \gamma} \\
f(\gamma)=0
\end{array}\right.
$$

(22) can be re-written as

$$
P_{e}^{s, r}=[u(\gamma) a Q(\sqrt{b \gamma})]_{\gamma_{a}}^{\gamma_{b}}+\frac{a \sqrt{b}}{2 \sqrt{2 \pi}} \int_{\gamma_{a}}^{\gamma_{b}} \frac{1}{\sqrt{\bar{\gamma}}} \exp \left(-\frac{b}{2} \gamma\right) d \gamma
$$

However note that the pdf range is $\gamma_{a}=\gamma_{0}$ and $\gamma_{b} \rightarrow \infty$. Substituting these limits in (25) and making the change of variable

$$
\left(\frac{b}{2}+\frac{1}{\bar{\gamma}}\right) \gamma=\frac{t^{2}}{2}
$$

we obtain the desired result given in (6).

\section{Derivation of the Constants $K_{i}$}

We prove in this appendix that

$$
K_{i}=\prod_{i^{\prime}=1, i^{\prime} \neq i}^{u} \frac{\gamma_{i}}{\gamma_{i}-\gamma_{i^{\prime}}}
$$

Without loss of generality, $K_{1}$ is derived here. The fractional expansion is equated to the product expression, i.e.

$$
\sum_{i^{\prime}=1}^{u} \frac{K_{i^{\prime}}}{1-\gamma_{i^{\prime}} s} \equiv \prod_{i^{\prime}=1}^{u} \frac{1}{1-\gamma_{i^{\prime}} s}
$$

To obtain $K_{1}$, (28) is multiplied by $\left(1-\gamma_{1} s\right)$ giving

$$
\left(1-\gamma_{1} s\right) \quad \sum_{i^{\prime}=1}^{u} \frac{K_{i^{\prime}}}{1-\gamma_{i^{\prime}} s} \equiv\left(1-\gamma_{1} s\right) \prod_{i^{\prime}=1}^{u} \frac{1}{1-\gamma_{i^{\prime}} s}
$$

after which $\mathrm{s}$ is set to $s=1 / \gamma_{1}$ yielding

$$
K_{1}=\prod_{i^{\prime}=2}^{u} \frac{1}{1-\frac{\gamma_{i^{\prime}}}{\gamma_{1}}}=\prod_{i^{\prime}=2}^{u} \frac{\gamma_{1}}{\gamma_{1}-\gamma_{i^{\prime}}}
$$

The same procedure is repeated for any $K_{i}$ in order to derive (27).

\section{REFERENCES}

[1] J. N. Laneman, D. N. Tse, and G. W. Wornell, "Cooperative diversity in wireless networks: Efficient protocols and outage behavior," IEEE Trans. Inform. Theory, vol. 50, no. 12, pp. 3062-3080, Dec. 2004.

[2] A. Sendonaris, E. Erkip, and B. Aazhang, "User cooperation diversity, part i:system description," IEEE Trans. Comm., vol. 51, no. 11, pp. 1927-1938, Nov. 2003.

[3] — - "User cooperation diversity, part ii:implementation aspects and performance analysis," IEEE Trans. Comm., vol. 51, no. 11, pp. 1939 1948, Nov. 2003.

[4] T. M. Cover and C. S. K. Leung, "An achievable rate region for the multiple-access channel with feedback," IEEE Transactions on Informations Theory, vol. IT-27, no. 3, pp. 292-298, May 1981.
[5] V. Emamian and M. Kaveh, "Combating shadowing effects for systems with transmitter diversity by using collaboration among mobile users," in Proc. Int. Symp. Communications, Taipei, Taiwan, Nov. 2001.

[6] A. Sendonaris, E. Erkip, and B. Aazhang, "Increasing uplink capacity via user cooperation diversity," in Proc. Int. Symp. Information Theory, Cambridge, MA, Aug. 1998.

[7] T. M. Cover and A. A. E. Gamal, "Capacity theorems for relay channel," IEEE Transactions on Informations Theory, vol. IT-25, no. 5, pp. 572584, September 1979

[8] M. O. Hasna and M. S. Alouini, "End-to-end performance of transmission systems with relays over rayleigh-fading channels," IEEE Transactions on Wireless Communications, vol. 2, no. 6, pp. 1126-1131, Nov. 2003.

[9] C. T. K. Ng and A. J. Goldsmith, "Capacity and power allocation for transmitter and receiver cooperation in fading channels," in Proc. IEEE International Conference on Communications.(ICC), Istanbul, Turkey, June 2006.

[10] P. A. Anghel, G. Leus, and M. Kaveh, "Distributed space-time cooperative systems with regenerative relays," IEEE Transactions on Wireless Communications, vol. 5, no. 11, pp. 3130-3140, Nov. 2006.

[11] C. T. K. Ng, N. Jindal, A. J. Goldsmith, and U. Mitra, "Capacity gain from two-transmitter and two-receiver cooperation," IEEE Transactions on Information Theory, 2007, to be published.

[12] J. N. Laneman and G. W. Wornell, "Distributed space-time-coded protocols for exploiting cooperative diversity in wireless networks," IEEE Trans. Inform. Theory, vol. 49, no. 10, pp. 2415-2425, Oct. 2003.

[13] M. Dohler, "Virtual antenna arrays," Ph.D. dissertation, University of London, United Kingdom, 2004.

[14] K. Tourki and L. Deneire, "End-to-end performance analysis of twohop asynchronous cooperative diversity," in 49th annual IEEE Global Telecommunications Conference, San Francisco, California, USA, Nov. 2006.

[15] T. E. Hunter, S. Sanayei, and A. Nosratinia, "Outage analysis of coded cooperation," IEEE Transactions on Information Theory, vol. 52, no. 2 , pp. 375-391, February 2006.

[16] P. Herhold, E.Zimmermann, and G. Fettweis, "A simple cooperative extension to wireless relaying," in Proc Int. Zurich Seminar on Commun.(IZS), pp. 3639., Zurich, Switzerland, 2004.

[17] S. M. Alamouti, "A simple transmit diversity technique for wireless communications," IEEE Journal on Select Areas in Communcations, vol. 16, no. 8, pp. 1451-1458, Oct. 1998.

[18] G. Scutari and S. Barbarossa, "Distributed space-time coding for regenerative relay networks," IEEE Transactions on Wireless Communications, vol. 4, no. 5, pp. 2387-2399, Sep. 2005.

[19] K. Tourki and L. Deneire, "Channel and delay estimation algorithm for asynchronous cooperative diversity," Springer's International Journal Wireless Personal Communications : Special Issue on Elective Topics towards Vision to Reality of the Wireless Future, vol. 37, no. 3-4, pp. 361-369, May 2006, invited paper.

[20] J. N. Laneman and G. W. Wornell, "Energy-efficient antenna sharing and relaying for wireless networks," in Proc. IEEE Wireless comm. and Networking Conf. (WCNC), Chicago, IL, USA, Sep. 2000.

[21] K. S. Marvin and M. S. Alouini., Digital Communication over Fading Channels, A Unified Approach to Performance Analysis. Wiley Series in Telecommunications and Signal Processing, 2000.

[22] H. Shin and J. H. Lee, "'"Exact Symbol Error Probability of Orthogonal Space-Time Block Codes"," in Proc. of the IEEE Global Telecommunications Conference., Taipei, Taiwan, Nov. 2002.

[23] K. Tourki and L. Deneire, "'Channel And Delay Estimation Algorithm For Asynchronous Cooperative Diversity"," in The 9th International Symposium on Wireless Personal Multimedia Communications, San Diego, CA, USA, Sep. 2006. 